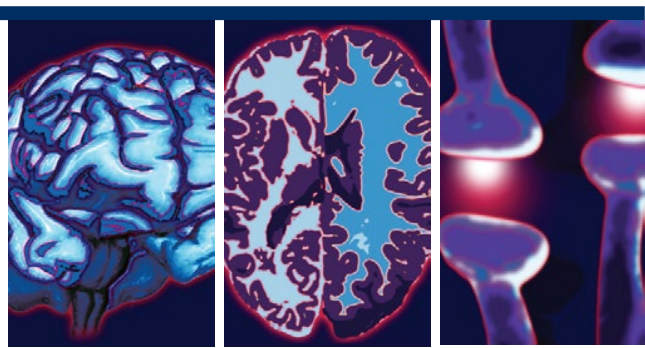

\title{
Age Effect on Cognition Improvements after Unilateral Anterior Temporal Lobectomy in Adults with Temporal Lobe Epilepsy
}

Qian Wang ${ }^{1,2}$, Feng Zhai ${ }^{2}$, Mengyang Wang³, Yuguang Guan², Jing Wang³, Yao Zhang², Hongchuan Niu², Guoming Luan ${ }^{1,2,4}$, Jian Zhou ${ }^{2,+}$

\begin{abstract}
Background

Patients with temporal lobe epilepsy (TLE) suffer from both uncontrollable seizures and cognition impairments. Although unilateral anterior temporal lobectomy (ATL) is one of the most efficient treatments for TLE, the cognition outcomes are still under debate. The purpose of the current study was to reveal whether the hemisphere of ATL and other clinical characters would affect the cognition outcomes in adults with TLE.
\end{abstract}

\section{Methods}

The neuropsychological and clinical data of 29 right and 24 left TLE patients who treated with unilateral ATL between February, 2014 and March, 2016 in a single epilepsy center were analyzed. Neuropsychological data were collected from preoperative, 3 and 12 months postoperative stages, including verbal intelligence quotient (VIQ), performance intelligence quotient $(\mathrm{PIQ})$, full intelligence quotient (FIQ), memory quotient (MQ), age of ATL surgery, age of seizure onset, and illness duration.

\section{Results}

The PIQ scores of the patients with left ATL were significantly improved while the MQ scores of the patients with right ATL was significantly improved, in both 3 and 12 months postoperative stages. Specially, the short-term memory scores of the patients with right ATL were significantly improved, in both 3 and 12 months postoperative stages. Significant negative correlations were found between memory improvements (especially short-term memory) and ages of both ATL surgery and ages of seizure onset in patients with left ATL.

\section{Conclusion}

The left ATL could mainly improve the performance intelligence while the right ATL could mainly improve the memory of patients with TLE. Either earlier ages of ATL surgery or ages of seizure onset could predict better memory outcomes after left ATL.

\section{Keywords:}

Temporal lobe epilepsy (TLE), Anterior temporal lobectomy (ATL), Memory, Performance Intelligence, Age of ATL surgery, Age of seizure onset

'Beijing Key Laboratory of Epilepsy, Sanbo Brain Hospital, Capital Medical University, Beijing, China 100093

${ }^{2}$ Department of Neurosurgery, Epilepsy Center, Sanbo Brain Hospital, Capital Medical University, Beijing, China 100093

3Department of Neurology, Epilepsy Center, Sanbo Brain Hospital, Capital Medical University, Beijing, China 100093

${ }^{4}$ Beijing Institute for Brain Disorders, Beijing, China 100069

${ }^{\dagger}$ Author for correspondence: Jian Zhou, M.D., Ph.D., Department of Neurosurgery, Epilepsy Center, Sanbo Brain Hospital, Capital Medical University, Beijing, China 100093. Tel: +86 010 62856910, Fax: +86 010 62856910, email: zhoujian5151@163.com 


\section{Introduction}

Epilepsy is defined as a neurological disorder characterized by recurrent and unpredictable interruptions of normal brain function, i.e. epileptic seizures [1]. Temporal lobe epilepsy (TLE) is the most common category of epilepsy [2]. For patients with medically intractable TLE, unilateral anterior temporal lobectomy (ATL), which is the surgical resection of the hippocampus, the amygdala, the temporal pole and the most anterior part of the temporal gyrus is an effective treatment [3-7].

Chronic TLE is associated with various cognition impairments [8-10]. A recent study reveals that the TLE patients had lower verbal intelligence quotient (VIQ), performance intelligence quotient (PIQ), and memory quotient (MQ) than nonTLE patients [8]. However, the cognition outcomes after unilateral ATL are still under debate. Some studies suggest cognition declines after ATL [11-14], some other studies reveal cognition improvements after ATL [1520].

Since more and more recent studies focus on the predictive factors of cognition outcomes [21,22], new opinions suggest that wide variability existed in the effects of ATL on postoperative memory function [23]. Various clinical characters have been suggested to explain cognitive change after temporal lobe surgery including age at onset, illness duration, seizure-free period, and frequency of seizure [24,25]. For example, a recent study suggested that age at surgery could predict the cognitive improvements because that the mean age of patients with memory improvement was significantly lower than those with memory decline [26]. On the other hand, some study also suggested memory outcomes were depended on the hemisphere, which indicated that patients with right hemisphere temporal lobe surgery usually had a better memory outcomes [26,27]. Therefore, the efforts made to predict which patients are at higher risk of experiencing better or worse cognition outcomes following ATL are essential.

In the current study, VIQ, PIQ, MQ scores were tested among 53 TLE patients from a single center who underwent unilateral ATL (29 left and 24 right) in preoperative and postoperative stages. Our purposes were (1) to evaluate the effects on cognition outcomes after left and right ATL and (2) to reveal whether clinical factors (age of ATL surgery, age of seizure onset, and illness duration) were associated with cognition outcomes after unilateral ATL.

\section{Methods}

\section{- Participants}

Totally 53 patients (29 left ATL and 24 right ATL) suffered from drug resistant temporal epilepsy and underwent unilateral anterior temporal lobectomy between February, 2014 and March, 2016 in Epilepsy Center of Sanbo Brain Hospital participated in this study. Twentynine patients (aged between 17 to 46 years, 11 females) suffered from left TLE and received the same side ATL while 24 patients (aged between 17 to 37 years, 15 females) suffered from left TLE and received the same side ATL (Table 1).

All patients were right-handed. Each of the patient participants underwent a standard ATL with the removal of the anterior temporal lobe, the amygdala, anterior half of the hippocampus, and/or the anterior one third of the parahippocampal gyrus. All patients provided written informed consent for their participation. All the patients participated in 3 month follow up. Twenty-two patients in left ATL group and twenty patients in right ATL group participated in 1 year follow up.

\section{- Neuropsychological testing}

Wechsler Adult Intelligence Scale - revised in China (WAIS-RC) [28,29] was used to measure the verbal intelligence quotient (VIQ) and performance intelligence quotient (PIQ) in the current study. Wechsler Memory Scale - revised in China (WMS-RC) [30,31] which include longterm memory subtest and short-term memory subtest was used to measure the memory quotient (MQ). According to the definition of Wechsler Scale, the standard scores of VIQ, PIQ and MQ were around 100 (> 100: better than the norm; < 100 , worse than the norm).

All patients were tested before and three months after operation. Twenty-two left TLE patients and 20 right TLE patients were tested twelve months after operation. All the neuropsychological evaluations were conducted by a well-trained psychologist and demographic and clinical data were collected by other medical doctors.

\section{Statistical analyses}

Statistical analyses were performed with IBM SPSS Statistics 20 (SPSS Inc., Chicago, Illinois 60606). Analyses of variances (ANOVAs), $t$-tests and Pearson correlation tests were conducted. The nullhypothesis rejection level was set at 0.05 . 


\section{Results}

\section{- Clinical characters}

The mean age of seizure onset was 13.10 years (standard deviation 10.18 years) in left ATL group and 9.38 years (standard deviation 7.04 years) in right ATL group. The mean illness duration was 15.76 years (standard deviation 9.82 years) in left ATL group and 14.63 years (standard deviation 6.55 years) in right ATL group. No significant difference was found in the age of onset $[t(51)=1.551, p=0.127]$, the age of ATL surgery $[t(51)=1.830, p=0.074]$, or the illness duration between $[t(51)=0.488, p$ $=0.628]$ between two groups.

\section{- Effect on seizure frequency}

Before ATL treatment, the average seizure frequency in left ATL group was as follows: < 10 seizures /month in 13 patients (44.8\%), 10100 seizures /month in 15 patients $(51.7 \%)$, and $>100$ seizures/month in 1 patients (3.5\%), while the average seizure frequency in right ATL group was as follows: < 10 seizures /month in 17 patients $(70.8 \%), 10-100$ seizures /month in 6 patients $(25.0 \%)$, and $>100$ seizures/month in 1 patients $(4.2 \%)$.

At 12 months follow-up, 79.3\% patients in left ATL group and $95.8 \%$ patients in right ATL group were seizure-free. All patients achieved > $50 \%$ seizure-frequency reduction compared with preoperative stage. All diagnosis and follow-up were ensured by pediatric neurologists in our epilepsy center.

\section{- Cognition improvements after left or right ATL}

To examine the operation effect and laterality effect, a 2 (operation effect: preoperative, postoperative) $\times 2$ (laterality effect: left-ATL, right-ATL) mixed two-way ANOVAs were conducted for VIQ, PIQ and MQ, respectively.

For 3 months after unilateral ATL, neither the operation effect $[F(1,51)=0.143, p=0.707]$ nor the laterality effect was significant $[F(1,51)$ $=0.431, p=0.515]$ in VIQ tests. In PIQ tests, the operation effect was significant $[F(1,51)=$ 8.516, $p=0.005]$ and the laterality effect $[F(1$, $51)=23.155, p=0.052]$ was not significant, but the interaction effect $[F(1,51)=0.172, p=$ $0.680]$. In MQ tests, the operation effect $[F(1$, $51)=2.939, p=0.093]$ and the laterality effect $[F(1,51)=3.229, p=0.078]$ was not significant, and the interaction effect was significant $[F(1$, $51)=5.975, p=0.018]$. Post hoc tests showed

\begin{tabular}{|} 
Table 1: Basic demographic and descriptive characteristics in both groups. \\
\begin{tabular}{|l|l|l|}
\hline & $\begin{array}{l}\text { Left ATL Group } \\
\text { (n=31) }\end{array}$ & $\begin{array}{l}\text { Right ATL Group } \\
\text { (n=24) }\end{array}$ \\
\hline Gender (male/female) & $20 / 11$ & $9 / 15$ \\
\hline Handedness (right/left) & $31 / 0$ & $24 / 0$ \\
\hline Age of seizure onset (years) & $13.10(10.18)$ & $9.38(7.04)$ \\
\hline Age of ATL surgery (years) & $28.86(6.74)$ & $24.00(5.07)$ \\
\hline Duration of illness (years) & $15.76(9.82)$ & $14.63(6.55)$ \\
\hline *mean (standard deviation) & & \\
\hline
\end{tabular}
\end{tabular}

a significantly improved PIQ in after left ATL surgery ( $p=0.031$, with Bonferroni adjustment), and a significantly improved MQ in after right ATL surgery $(p=0.008$, with Bonferroni adjustment) (Figure 1, left panels).

For 12 months after unilateral ATL, neither the operation effect $[F(1,51)=0.417, p=0.552]$ nor the laterality effect was significant $[F(1,51)$ $=1.901, p=0.176]$ in VIQ tests. In PIQ tests, the operation effect was significant $[F(1,51)=$ 9.174, $p=0.004]$ but the laterality effect $[F(1$, $51)=0.379, p=0.542]$ and the interaction effect $[F(1,51)=0.576, p=0.452]$ was not significant. In MQ tests, the laterality effect $[F(1,51)=$ 3.663, $p=0.063]$ was not significant, but the operation effect $[F(1,51)=2.208, p=0.145]$ and the interaction effect was not significant $[F$ $(1,51)=1.767, p=0.191]$. Post hoc tests showed a significantly improved PIQ in after left ATL surgery ( $p=0.021$, with Bonferroni adjustment), and a significantly improved MQ in after right ATL surgery $(p=0.037$, with Bonferroni adjustment) (Figure 1, right panels).

\section{- Memory improvements after left or right ATL}

To examine the operation effect and laterality effect, a 2 (operation effect: preoperative, postoperative) $\times 2$ (laterality effect: left-ATL, right-ATL) mixed two-way ANOVAs were conducted for long-term and short-term memory, respectively.

For 3 months after unilateral ATL, neither the operation effect $[F(1,51)=1.892, p=0.175]$ nor the laterality effect $[F(1,51)=0.706, p=$ 0.405 ] was significant in long-term memory tests. But in short-term memory tests, both the operation effect $[F(1,51)=7.038, p=0.011]$ and the laterality effect $[F(1,51)=8.195, p=$ $0.006]$ was significant, the interaction effect was not significant $[F(1,51)=0.248, p=0.620]$. Post hoc tests showed a significantly improved short-term memory in after right ATL surgery ( $p=0.018$, with Bonferroni adjustment $)$ (Figure 2, left panels). 


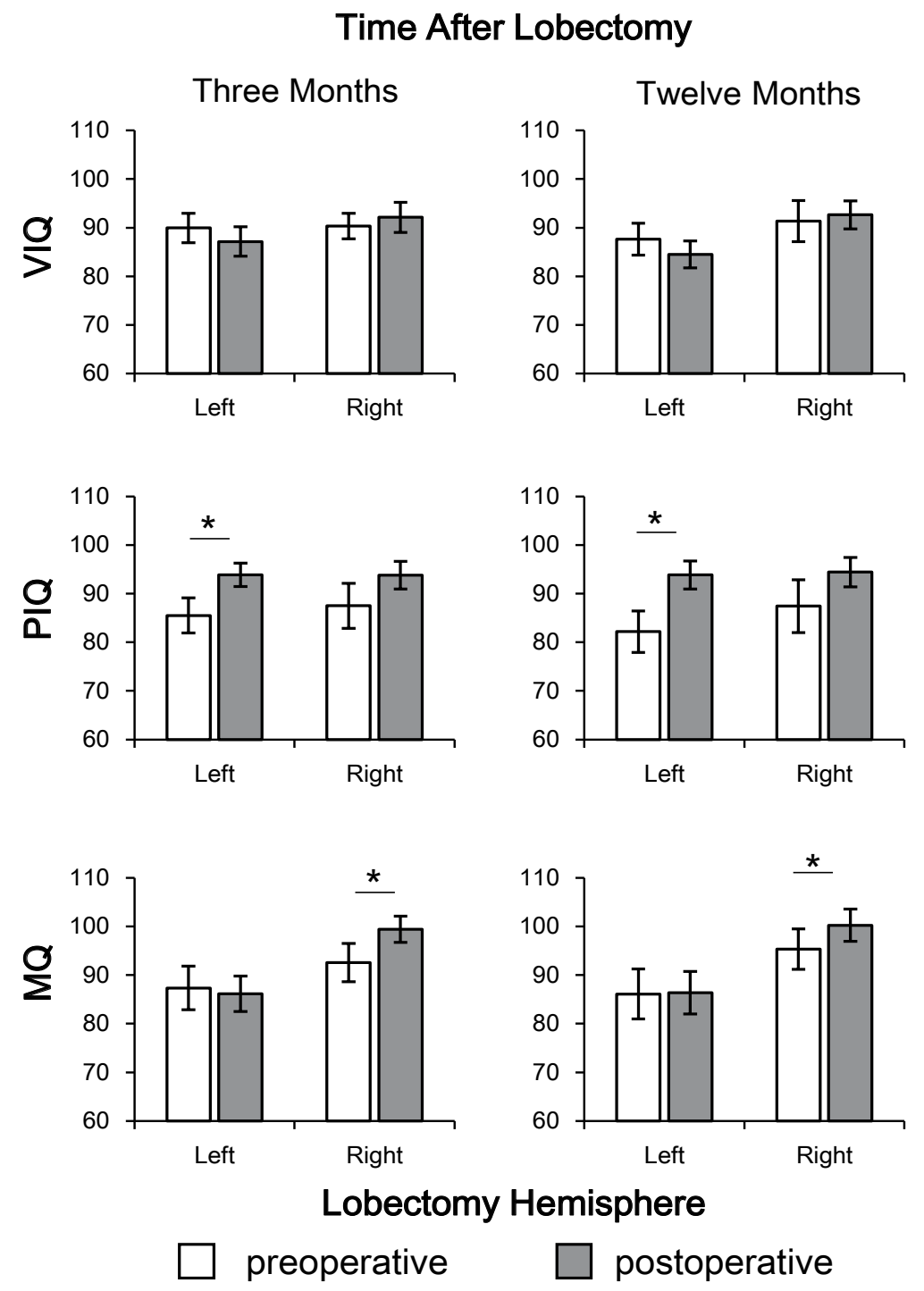

Figure 1: Comparisons of VIQ, PIQ, MQ scores between preoperative and postoperative stages in left and right TLE patients, respectively. Left panels: cognition scores before and three months after unilateral ATL. Right panels: cognition scores before and twelve months after unilateral ATL. VIQ: verbal intelligence quotient; PIQ: performance intelligence quotient; $M Q$ : memory quotient; TLE: temporal lobe epilepsy; $A T L$ : anterior temporal lobectomy.

${ }^{*}, p<0.05$.

\section{- Correlation between cognition improvements and clinical characters}

To test whether cognition improvements after unilateral ATL were correlated with clinical characters of TLE patients, Pearson correlation tests were conducted between changes of cognition indices (VIQ, PIQ and MQ) and clinical indices (age of ATL surgery, age of seizure onset, and illness duration). Significant negative correlations were only found between MQ and both age of ATL surgery $(r=-0.442$, $p=0.016)$ and age of seizure onset $(r=-0.491$, $p=0.007$ ) three months after ATL (Figures 3 and 4). No significant correlations were found between any cognition improvement and illness duration (all $p>0.05)$.

To further test whether memory improvements after unilateral ATL were correlated with clinical characters of TLE patients, Pearson correlation tests were conducted between changes of memory indices (long-term and short-term memory) and clinical indices (age of ATL surgery, age of seizure onset, and illness duration). Significant negative correlations were only found between short-term memory and both age of ATL surgery $(r=-0.406, p=0.029)$ and age of seizure onset $(r$ $=-0.394, p=0.034)$ three months after ATL. No significant correlation was found in long-term memory (Figure 4). No significant correlation was found between any memory improvement and illness duration (all $p>0.05$ ). These results suggested that the earlier ATL surgery was conducted, the better memory improvements, especially the short-term improvements would be observed. On the other hand, the earlier of the seizure onset would possibly predict a better short-term memory enhancement after ATL surgery.

\section{Discussion}

In the current study, neuropsychological tests were conducted among 53 TLE patients from single center who underwent unilateral ATL (29 left and 24 right) to evaluate cognition outcomes in postoperative stages compared with those in preoperative stage. The main findings include: (1) the PIQ of the patients with left ATL was significantly improved while the MQ of the patients with right temporal lobe resection was significantly improved, in both 3 and 12 months postoperative stages; (2) the short-term memory scores of the patients with right ATL was significantly improved, in both 3 and 12 months postoperative stages; (3) Significant 
negative correlations were found between shortterm memory improvements and ages of both ATL surgery and seizure onset in patients with left ATL.

\section{- PIQ improvements after left ATL}

PIQ is significantly impaired in TLE patients [8]. However, there are few studies discussed about how PIQ in TLE patients would change after unilateral ATL in the literature. Lacruz et al. [32] suggested that the changes of PIQ after temporal lobectomy is associated with the contralateral Wada scores, which estimate cognitive function of the hemisphere that remains after surgery. In the current study, we observed a significant improvement of PIQ after left ATL, but not after right ATL, implying a possible function release from the non-dominant hemisphere with the disappearance of seizures.

\section{- Memory changes after unilateral ATL}

In the current study, a group difference was observed that right ATL significantly improved the MQ but left ATL failed. Previous studies also suggested worse memory outcomes after left ATL than after right ATL, while many patients who undergo left ATL show no change or might even show postoperative improvement [17-19], which is consistent with our results.

The total MQ score include long-term and short-term memory subtests. Some previous studies suggested that the long-term memory did not change after unilateral ATL, which is mainly associated with the postoperative seizure outcomes [33]. In the current study, the longterm memory scores showed no significant difference between pre and postoperative stages but the short-term memory scores were significantly improved after right ATL.

The neural connection between the auditory areas and medial temporal cortex (which comprises the hippocampal formation and the parahippocampal region) is involved in both long-term auditory memory [34] and shortterm auditory episodic memory [35]. A recent functional magnetic resonance imaging (fMRI) study suggested that that compensatory posterior hippocampal reorganization might be the underlying neural mechanisms of the memory improvement after right ATL [36]. Significant left posterior hippocampal activation that was sustained 3 and 12 months postoperatively during memory tasks, which is in accordance with our results that short-term memory improved in both 3 and 12 months postoperative stages.

\section{Time After Lobectomy}

Three Months
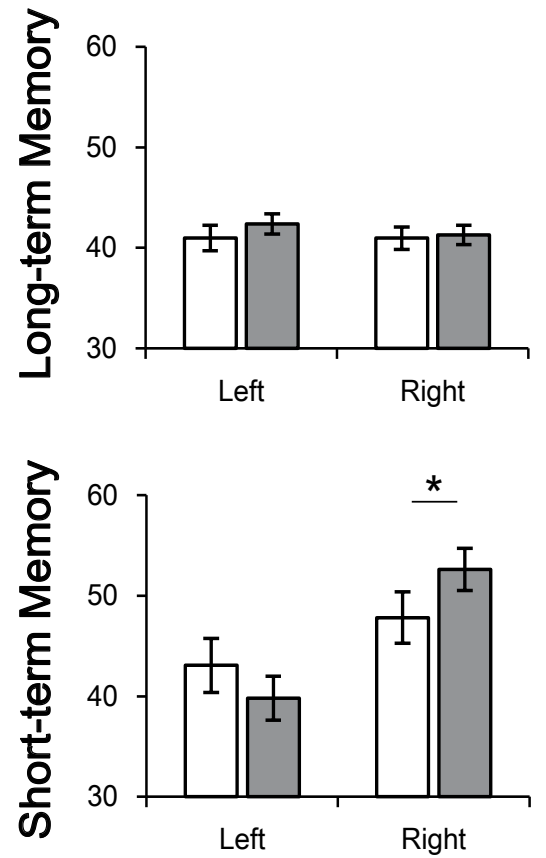

Lobectomy Hemisphere
Figure 2: Comparisons of long-term and short-term memory scores between preoperative and postoperative stages in left and right TLE patients, respectively. Left panels: memory scores before and three months after unilateral ATL. Right panels: memory scores before and twelve months after unilateral ATL. TLE: temporal lobe epilepsy; ATL: anterior temporal lobectomy.

$*, p<0.05$.

\section{- Correlation between memory outcomes and age}

Cognitive prognosis is poor for a subset of patients characterized by chronicity of epilepsy, older age, lower intellectual ability, and more baseline abnormalities in quantitative magnetic resonance volumetric [17].

In the group level, no significant difference was observed between preoperative and postoperative stages after left ATL. However, correlation tests showed a significant negative correlation between memory improvements and both ATL surgery and seizure onset (Figure 3 and 4). These results implied that if a patient with earlier age of seizure onset and earlier ATL treatment, the memory outcomes after left ATL would be better.

For that the left hemisphere is dominant, could these changes be linked to ipsilateral anatomical structure outside the epileptic zone? A recent 
Research Jian Zhou

A

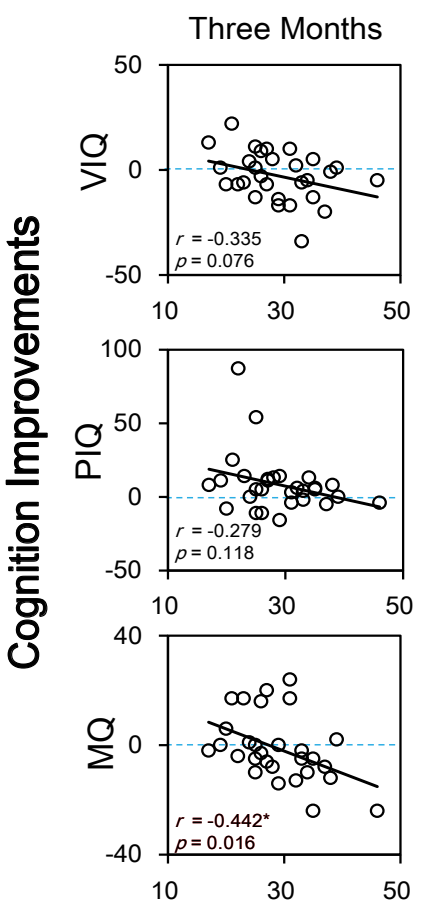

Time After Right ATL
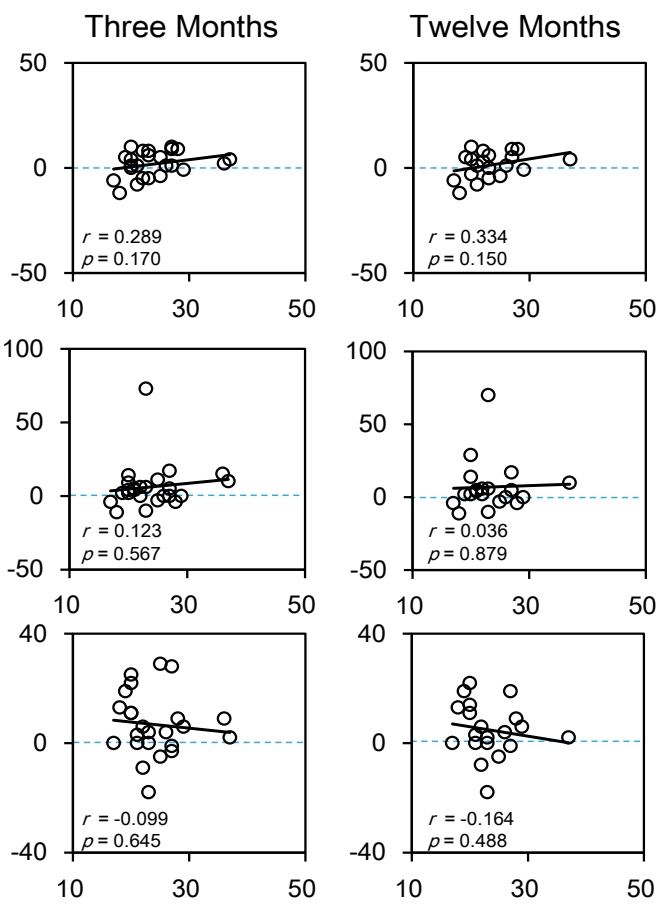

Age of ATL Surgery (years)

B

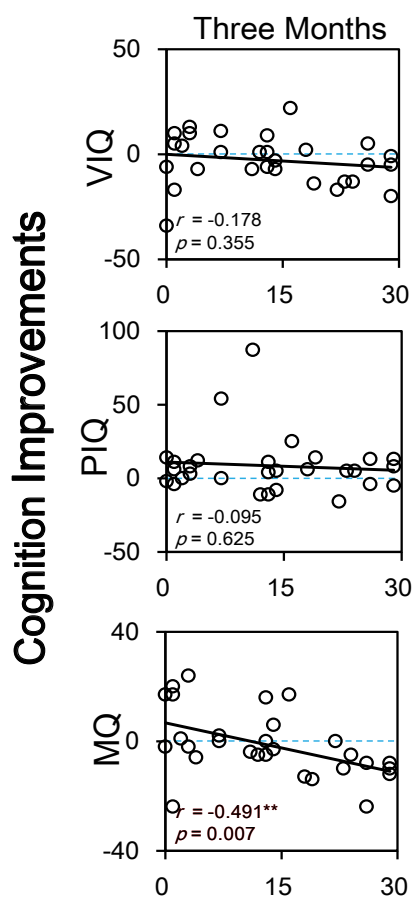

Time After Right ATL
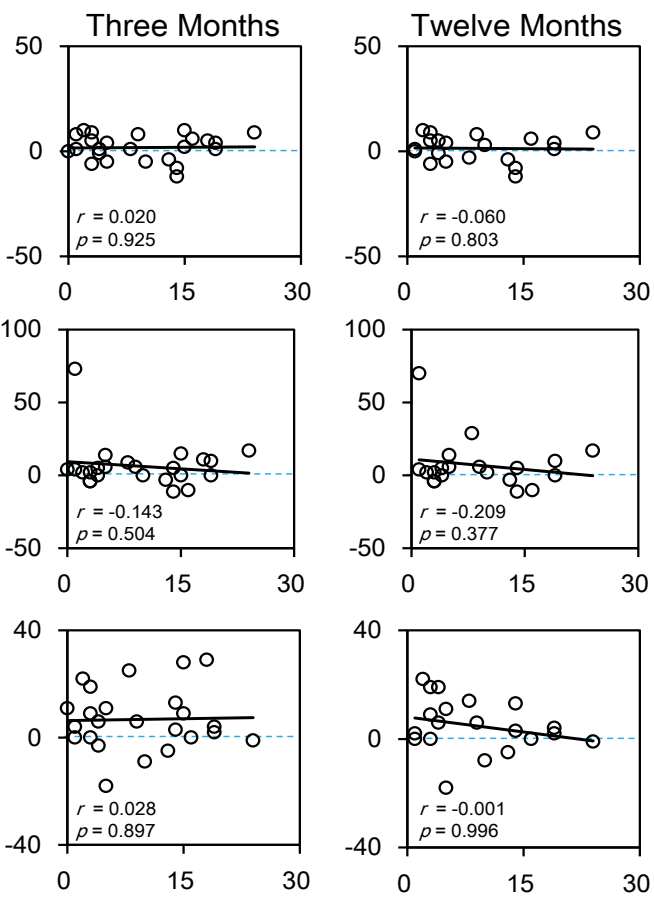

Age of Seizure Onset (years)

Figure 3: Examination of the correlation between the cognition improvements (VIQ, PIQ, MQ) and (A) age of ATL surgery and (B) age of seizure onset in left and right TLE patients, respectively. VIQ: verbal intelligence quotient; PIQ: performance intelligence quotient; $M Q$ : memory quotient; TLE: temporal lobe epilepsy.

${ }^{*}, p<0.05$. ${ }^{* *}, p<0.01$. 
Age Effect on Cognition Improvements after Unilateral Anterior Temporal Lobectomy in Adults with Temporal Lobe Epilepsy

A

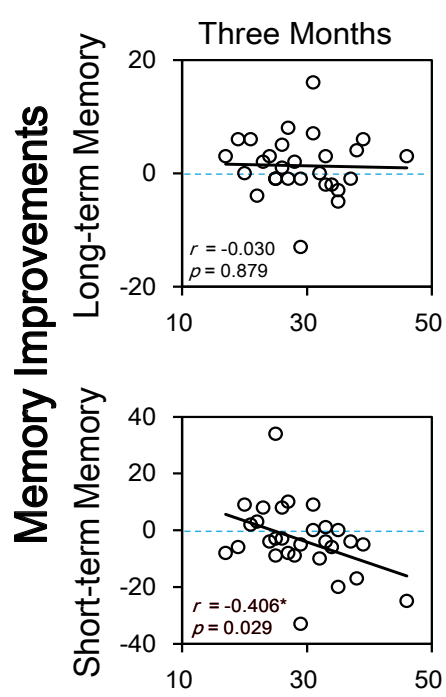

Time After Left ATL
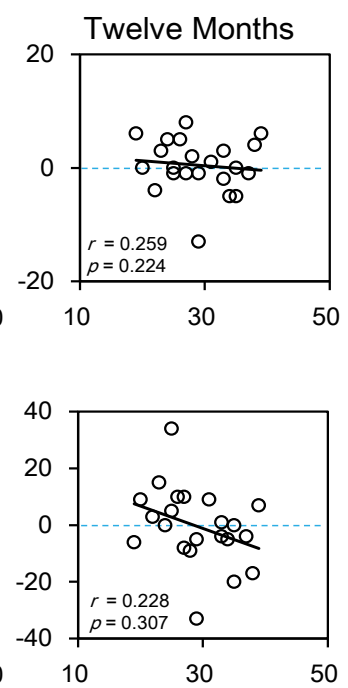

Time After Right ATL
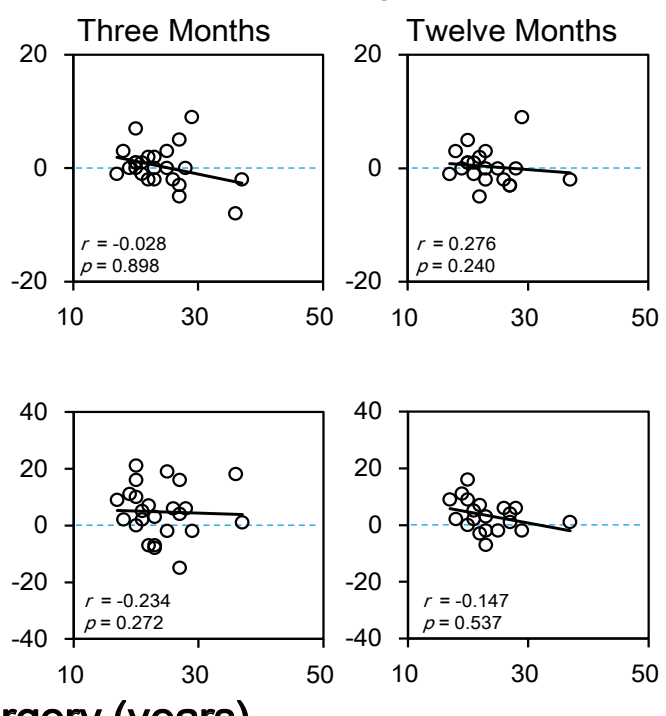

Age of ATL Surgery (years)

B

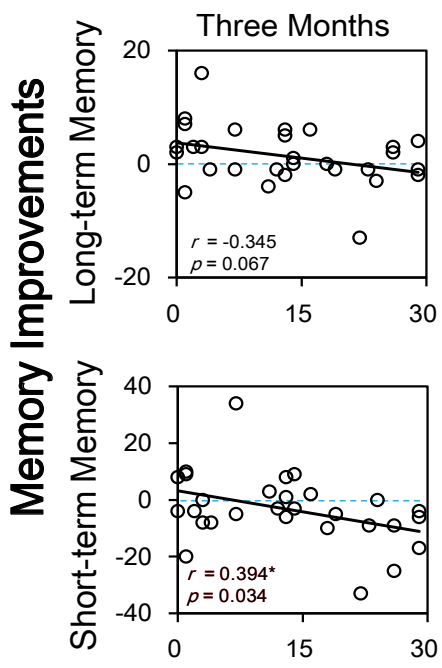

Time After Left ATL
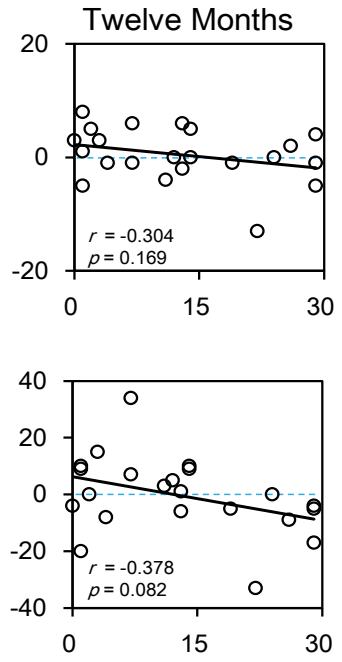

Time After Right ATL
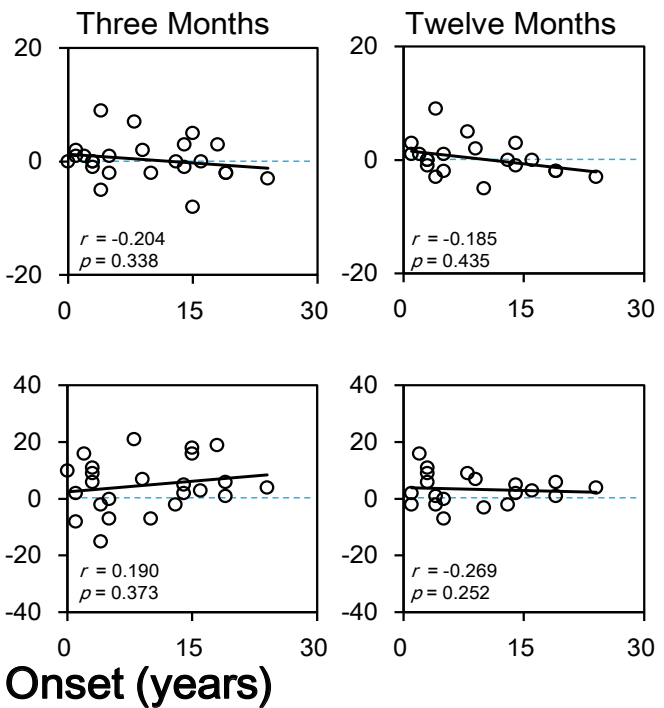

Age of Seizure Onset (years)

Figure 4: Examination of the correlation between the memory improvements (long-term memory and short-term memory) and (A) age of ATL surgery and (B) age of seizure onset in left and right TLE patients, respectively. TLE: temporal lobe epilepsy.

${ }^{*}, p<0.05$.

functional magnetic resonance imaging (fMRI) revealed that patients with TLE had greater activation in the left posterior medial temporal lobe during memory tasks postoperatively than preoperatively [15], suggesting that it is the capacity of the posterior remnant of the ipsilateral hippocampus rather than the functional reserve of the contralateral hippocampus that is important for maintaining memory function after unilateral ATL.
In a recent study suggested, Cano-Lopez et al. [26] also discussed the relationship between cognition out comes and age at surgery. In both Cano-Lopez's study and the current study, patients with right hemisphere temporal lobe surgery had better memory outcomes than those in left hemisphere group. More importantly, in Cano-Lopez's study, the conclusion that age at surgery could predict the cognitive improvements was generalized from 
that the mean age of patients with memory improvement was significantly lower than those with memory decline. However in the current study, we proposed a similar conclusion based on the Pearson correlation tests, which was more stringent to reach the significance level and further suggested a continuous age effect of the time at ATL surgery. What's more, the CanoLopez's study didn't separate the left and right ATL groups in age at surgery prediction, while the current study clearly revealed that the age of ATL surgery was only negatively correlated with memory scores in the left ATL group.

\section{Limitations}

Although the current study gave a new insight of age effect on unilateral ATL treatments, there were still some shortcomings for the retrospective approach. For instance, this study did not include data regarding the educational level, or drug use (smoking), or parental education, which could be related to differences in intelligence performance. On the other hand, it lacks a control group of patients with refractory epilepsy not surgically-treated and it also has a limited number of patients. Future studies should consider these factors.

\section{Conclusion}

The left temporal lobe resection could mainly improve the performance intelligence while the right temporal lobe resection could mainly improve the memory of patients with temporal lobe epilepsy. Either earlier ages of ATL surgery or ages of seizure onset could predict better memory outcomes after left ATL, which mainly suggested the importance of early surgical treatment.

\section{Acknowledgements}

This work was supported by the Beijing Municipal Science \& Technology Commission (Z161100000116085), National Natural Science Foundation of China (81671285), the China Postdoctoral Science Foundation (2016M601066), the Capital Health Research and Development of Special (2016-1-8012).

\section{References}

1. Fisher RS, Boas WE, Blume W, et al. Epileptic seizures and epilepsy: definitions proposed by the International League Against Epilepsy (ILAE) and the International Bureau for Epilepsy (IBE). Epilepsia 46(4), 470-472 (2005).

2. Tellez-Zenteno JF, Hernandez-Ronquillo L. A review of the epidemiology of temporal lobe epilepsy. Epilepsy. Res. Treat 2012(1), 630853 (2012).

3. Radhakrishnan K, So EL, Silbert PL, et al. Predictors of outcome of anterior temporal lobectomy for intractable epilepsy $A$ multivariate study. Neurology 51(2), 465-471 (1998).

4. Wiebe S, Blume WT, Girvin JP, et al. A randomized, controlled trial of surgery for temporal-lobe epilepsy. N. Engl. J. Med 345(5), 311-318 (2001).

5. Cross JH, Duchowny M. Transition in lesional focal epilepsy, and following epilepsy surgery. Epilepsia 55(s3), 34-36 (2014).

6. Jehi LE, Palmini A, Aryal U, et al. Cerebral cavernous malformations in the setting of focal epilepsies: pathological findings, clinical characteristics, and surgical treatment principles. Acta. Neuropathol 128(1), 55-65, (2014).

7. Jobst BC, Cascino GD. Resective epilepsy surgery for drug-resistant focal epilepsy: a review. JAMA 313(3), 285-293 (2015).
8. Guo L, Bai G, Zhang H, et al. Cognitive Functioning in Temporal Lobe Epilepsy: A BOLD-fMRI Study. Mol. Neurobiol 1-9 (2016).

9. Helmstaedter C, Kurthen M, Lux S, et al. Chronic epilepsy and cognition: a longitudinal study in temporal lobe epilepsy. Ann. Neurol 54(4), 425-432 (2003).

10. Zheng J, Qin B, Dang C, et al. Alertness network in patients with temporal lobe epilepsy: a fMRI study. Epilepsy. Res 100(1), 67-73 (2012).

11. Hermann BP, Seidenberg M, Haltiner A, et al. Relationship of age at onset, chronologic age, and adequacy of preoperative performance to verbal memory change after anterior temporal lobectomy. Epilepsia 36(2), 137-145, (1995).

12. Helmstaedter C, Petzold I, Bien CG. The cognitive consequence of resecting nonlesional tissues in epilepsy surgery-Results from MRI-and histopathology-negative patients with temporal lobe epilepsy. Epilepsia 52(8), 1402-1408 (2011).

13. Liu Z, Wang Q, You Y, et al. The role of the temporal pole in modulating primitive auditory memory. Neurosci. Lett 619(1), 196202 (2016).

14. Mansouri A, Fallah A, McAndrews MP, et al. Neurocognitive and seizure outcomes of selective amygdalohippocampectomy versus anterior temporal lobectomy for mesial temporal lobe epilepsy. Epilepsy. Res. Treat

\section{2 (2014).}

15. Bonelli SB, Thompson PJ, Yogarajah M, et al. Memory reorganization following anterior temporal lobe resection: a longitudinal functional MRI study. Brain 105 (2013).

16. Hermann BP, Wyler AR. Effects of anterior temporal lobectomy on language function: a controlled study. Ann. Neurol 23(6), 585-588 (1988).

17. Hermann BP, Seidenberg M, Dow $C$, et al. Cognitive prognosis in chronic temporal lobe epilepsy. Ann. Neurol 60(1), 80-87 (2006).

18. Morino M, Uda T, Naito K, et al. Comparison of neuropsychological outcomes after selective amygdalohippocampectomy versus anterior temporal lobectomy. Epilepsy. Behav 9(1), 95100 (2006).

19. Powell GE, Polkey CE, McMillan T. The new Maudsley series of temporal lobectomy. I: Short-term cognitive effects. Br. J. Clin. Psychol 24(2), 109-124 (1985).

20. Jokeit $\mathrm{H}$, Ebner A. Long term effects of refractory temporal lobe epilepsy on cognitive abilities: a cross sectional study. J. Neurol. Neurosurg. Psychiatry 67(1), 44-50 (1999).

21. Law N, Benifla M, Rutka J, et al. Verbal memory after temporal lobe epilepsy surgery in children: Do only mesial structures matter? Epilepsia 58(2), 291-299 (2017).

22. Giovagnoli AR, Parente A, Didato G, et al. The 
Age Effect on Cognition Improvements after Unilateral Anterior Temporal Lobectomy in Adults with Temporal Lobe Epilepsy

course of language functions after temporal lobe epilepsy surgery: a prospective study. Eur. J. Neurol 23(12), 1713-1721 (2016).

23. Bell B, Lin JJ, Seidenberg M, et al. The neurobiology of cognitive disorders in temporal lobe epilepsy. Nat. Rev. Neurol 7(3), 154-164 (2011)

24. Ji GJ, Zhang Z, Xu Q, et al. Connectome reorganization associated with surgical outcome in temporal lobe epilepsy. Medicine 94(40), e1737 (2015).

25. Sidhu MK, Stretton J, Winston GP, et al. Factors affecting reorganisation of memory encoding networks in temporal lobe epilepsy. Epilepsy. Res 110(1), 1-9 (2015).

26. Cano-López I, Vázquez JF, Campos A, et al. Age at surgery as a predictor of cognitive improvements in patients with drug-resistant temporal epilepsy. Epilepsy. Behav 70(A), 1017 (2017).
27. Baxendale S, Thompson P, Harkness W, et al. Predicting memory decline following epilepsy surgery: a multivariate approach. Epilepsia 47(11), 1887-1894 (2006).

28. Wechsler D. WAIS-R manual: Wechsler adult intelligence scale-revised. Psychological Corporation (1981)

29. Gong Y X. Manual of Wechsler Adult Intelligence Scale-Chinese Version. Changsha: Chinese Map (1992).

30. Wechsler D, Stone C P. Wechsler memory scale-revised. Psychological Corporation (1987).

31. Gong YX. Wechsler memory scale-revised in China. Hunan Medical University Publishing, Hunan (1989).

32. Lacruz ME, Alarcon G, Akanuma N, et al. Neuropsychological effects associated with temporal lobectomy and amygdalohippocampectomy depending on Wada test failure. J. Neurol. Neurosurg. Psychiatry 75(4), 600-607 (2004).

33. Evans SJ, Elliott G, Reynders H, et al. Can temporal lobe epilepsy surgery ameliorate accelerated long-term forgetting? Neuropsychologia 53(1), 64-74 (2014).

34. Engelien A, Stern E, Isenberg N, et al. The Parahippocampal Region and AuditoryMnemonic Processing. Ann. N Y Acad. Sc 911(1), 477-485 (2000).

35. Fritz J, Mishkin M, Saunders RC. In search of an auditory engram. Proceedings of the National Academy of Sciences of the United States of America 102(26), 9359-9364 (2005).

36. Sidhu MK, Stretton J, Winston GP, et al. Memory network plasticity after temporal lobe resection: a longitudinal functional imaging study. Brain 139(2), 415-430 (2016) 